\title{
Analysis of Relative Motion between Femoral Head and Acetabular Cup and Advances in Computation of the Wear Factor for the Prosthetic Hip Joint
}

O. Calonius, V. Saikko

The amount and type of wear produced in the prosthetic hip joint depends on the type of relative motion between the femoral head and the acetabular cup. Wear particles removed from the bearing surfaces of the joint can cause adverse tissue reactions resulting in osteolysis and ultimately in loosening of the fixation of the implant. When designing a simulator for evaluation of prospective materials for artificial hip joints it is important to verify that the type of relative motion at the articulation is similar to that produced in walking, involving continually changing direction of sliding. This paper is an overview of recent research done at Helsinki University of Technology on the analysis of the relationship between relative motion and wear in the prosthetic hip joint.

To analyze the relative motion, software for computing tracks, referred to as slide tracks, drawn on the counterface by marker points on the bearing surface was developed and experimentally verified. The overall relative motion of the joint was illustrated by a slide track pattern, produced by many points. The patterns resulting from walking motion and from motion produced in ten contemporary hip simulator types were compared. The slide track computations were not limited to illustrational purposes but offered a basis for computing variations of sliding distances, sliding speeds and direction of sliding during a cycle. This was done for the slide track termed the force track, drawn by the resultant contact force. In addition, the product of the instanlaneous load and increment of sliding distance was numerically integrated over a cycle. This track integral of load had so far not been determined for the majority of contemporary hip simulators. The track integral can be used in determining the wear factor, making it possible to compare clinical wear rates with those produced by hip simulators. The computation of the wear factor was subsequently improved by replacing the track integral of the resultant contact force with a surface integral computed as the sum of track integrals of a large number of smaller normal forces obtained by discretizing the contact pressure distribution. The slide track software could also be utilized in the conceptual design of new simulators because it was possible to rapidly investigate the effect of changes to the motion waveform amplitudes or phases, or of omitting certain waveforms to simplify the design of a simulator.

The slide track analysis showed that walking motion produced mainly open tracks on the center of contact, implying continually changing direction of sliding. This phenomenon, which is crucial for obtaining the correct wear mechanisms for acetabular cups made of polyethylene, was reproduced by simulators having abduction-adduction motion in addition to flexion-extension motion. In the force track computations involving contemporary simulators with the common femoral head size of $28 \mathrm{~mm}$, the sliding distance per cycle and the force track integral per cycle ranged from 19.7 to $34.3 \mathrm{~mm}$ and from 17.4 to $43.5 \mathrm{~N} \mathrm{~m}$, respectively. The average sliding speed ranged from 19.7 to $49.0 \mathrm{~mm} / \mathrm{s}$. The sum of track integrals computed with forces obtained by discretizing the contact pressure distribution reached a substantially higher value than the track integral obtained with the resultant contact force only. This suggests that the wear factor is actually overestimated when computed in the conventional way by dividing the wear rate with the force track integral.

Keywords: prosthetic hip joint, relative motion, slide track, wear simulators, wear factor.

\section{Notation}

Area of articulating surface, in HUT-BRM area of hemisphere $=2 \pi r^{2}$

$A_{\mathrm{E}} \quad$ Area of surface element $=A / N_{\mathrm{E}}$

$f(\theta) \quad$ Shape function of contact pressure distribution

$i \quad$ Time index $=$ index to points of discretized motion waveforms; $i=1,2,3, \ldots, N$

Surface integral replacing $\int L \mathrm{~d} x=$ sum of track integrals of discrete forces $q_{m}$

$k \quad$ Wear factor; $k=(V / n) / \int L \mathrm{~d} x$

$L \quad$ Joint contact resultant force

$m$ Index to surface elements and discrete forces; $m=1, \ldots, N_{\mathrm{E}}$

$n \quad$ No. of cycles

$N \quad$ No. of time steps per cycle $=$ no. of discrete rotation angles
$N_{\mathrm{E}} \quad$ No. of surface elements $=$ no. of discrete forces

$p \quad$ Contact pressure

$p_{\max }$ Peak value of contact pressure

$q_{m} \quad$ Discrete force corresponding to pressure load on surface element

$r \quad$ Radius of spherical articulating surface

r Position vector of surface element

$t \quad$ Time

$T \quad$ Cycle time

$v_{\text {mean }}$ Mean sliding velocity $=x_{L} / T$

V Wear volume

$x \quad$ Sliding distance; track coordinate

$x_{L} \quad$ Length of force track

$X Y Z$ Coordinate system (fixed), attached to center point of acetabular cup

$x y z \quad$ Coordinate system (moving), attached to center point of femoral head 
$\theta \quad$ Angle between $\mathbf{r}$ and $Z$-axis

$\xi \quad$ Direction of sliding relative to the acetabular cup

AA Abduction-adduction

AR Aspect ratio

Aspect ratio Major dimension divided by minor dimension of slide track

BRM Biaxial rocking motion hip wear simulator

CTPOD Circularly translating pin-on-disk wear test device

$\mathrm{FE}$

Force track Track made on the counterface by the point of joint contact resultant force

HUT-3 Helsinki University of Technology hip joint simulator Mark III

HUT-BRM Helsinki University of Technology BRM simulator

IER Internal-external rotation

In vivo Within the living organism

Slide track Track made on the counterface by point on surface of femoral head or acetabular cup

Track integral Line integral of normal force over its slide track

\section{Introduction}

Total hip joint replacement arthroplasty can provide the patient with dramatically improved quality of life by relieving pain and offering increased mobility. A failed prosthesis however causes pain, reduces the ability to work and necessitates a revision operation. The most common reason for revision operations is loosening of the components ( $66 \%$ of hip revisions in Finland [1]). The current understanding is that loosening is primarily due to bone loss around the implant, caused by adverse tissue reactions to wear particles $[2,3,4]$. Tribological research of prosthetic joints deals with friction, wear and lubrication of the implant with the purpose of increasing its lifetime. A particular area within this field of research is the design and development of wear simulation equipment for pre-clinical evaluation of prosthetic joint materials.

Wear simulation is done under conditions that are in a tribological sense close to the conditions in vivo. This means that the essential features of the relative motion at the articulation, the lubrication and the loading, are taken into consideration when setting up the wear simulation equipment. Monitoring of the simulation involves measuring the amount of worn material, visual and microscopic inspection of the wear surfaces and microscopic analysis of the wear debris. The results are validated against results of in vivo radiological wear measurements, or by comparing with results of inspection of components retrieved at revision operations or autopsy. Many studies have shown that only multidirectional motion yields realistic wear for polyethylene, which is a common material for acetabular cups in prosthetic hip joints $[5,6$, $7,8]$. Multidirectional motion means that the direction of sliding changes many times during the motion cycle, unlike, e.g., the situation in a regular pin-on-disk wear test device, in which the relative motion is unidirectional. Since the relative motion of the articulating surfaces is known to influence the production of wear debris $[6,7,9]$ and since the effect of wear particles on implant failure due to loosening is known, analysis of relative motion must be considered an important part of the development of wear simulation equipment. Motion analysis is preferably done during the simulator design stage, but can be applied in the evaluation of an existing simulator design, e.g., if the simulator does not reproduce the in vivo wear process.

This paper is an overview of research activities at Helsinki University of Technology (HUT) in the field of biotribology discussing the results and implications of recent articles dealing with the relationship between the relative motion of the articulating surfaces and wear in the prosthetic hip joint. Examples of other biotribology research activities at HUT include studies on the effect of lubricant on the wear of prosthetic joint materials $[10,11]$ and wear of crosslinked polyethylene [12, 13] and alumina [14]. The main objective of the research presented in this paper was to provide a quantitative basis for studies on the relationship between the type of relative motion and wear. The wear factor $k=(V / n) / \int L \mathrm{~d} x$ relates the wear rate to the load and the sliding distance. There are substantial differences between patients regarding load, extent of motion and number of gait cycles per year [15, $16,17,18]$. By computing the wear factor, these aspects can be taken into account to quantitatively compare the wear generated in a hip joint simulator with the wear produced in the human body [15] or with another wear test device. In contrast, the conventional wear rate (expressed as $\mathrm{mg}$ or $\mathrm{mm}^{3}$ per million cycles) is not well suited for making such comparisons because the aspects mentioned above are not taken into account.

\section{Methods}

The cyclic relative motion of the hip joint can be illustrated by computing tracks, known as slide tracks, made on the counterface by marker points on the surface of the femoral head or acetabular cup. When referring to the motion of the hip joint, the directions are named according to Fig. 1, which is a frontal view of the right hip joint. To compute the relative motion, two coordinate systems were placed at the mutual center of the cup and the head. The reference coordinate system $X Y Z$ was fixed relative to the cup and the moving coordinate system $x y z$ was fixed relative to the head. Initially the systems coincided with the $X$ and $x$ axes pointing in the medial, the $Y$ and $y$ axes in the posterior and the $Z$ and $z$ axes in the superior directions. The equatorial plane of the cup, and initially the equatorial plane of the head, was perpendicular to the coronal plane. The values of the cup angle, the head axis angle and the force angle in the coronal plane depended on the simulated case ([29], Table 1).

The motion of a marker point was based on Euler rotations with the axes of the moving coordinate system acting as rotation axes according to the sequence determined for each simulation. See Fig. 2 for an example of applying the rotation sequence $\mathrm{FE} \rightarrow \mathrm{AA} \rightarrow \mathrm{IER}$. To compute a cup track the marker point was fixed to the head, and to compute a head track the marker point was fixed to the cup. The cyclic $\mathrm{FE}, \mathrm{AA}$ and IER rotation angles, i.e., the motion waveforms applied to the axes were taken from a biomechanical study [19] and from published data of different simulator designs 


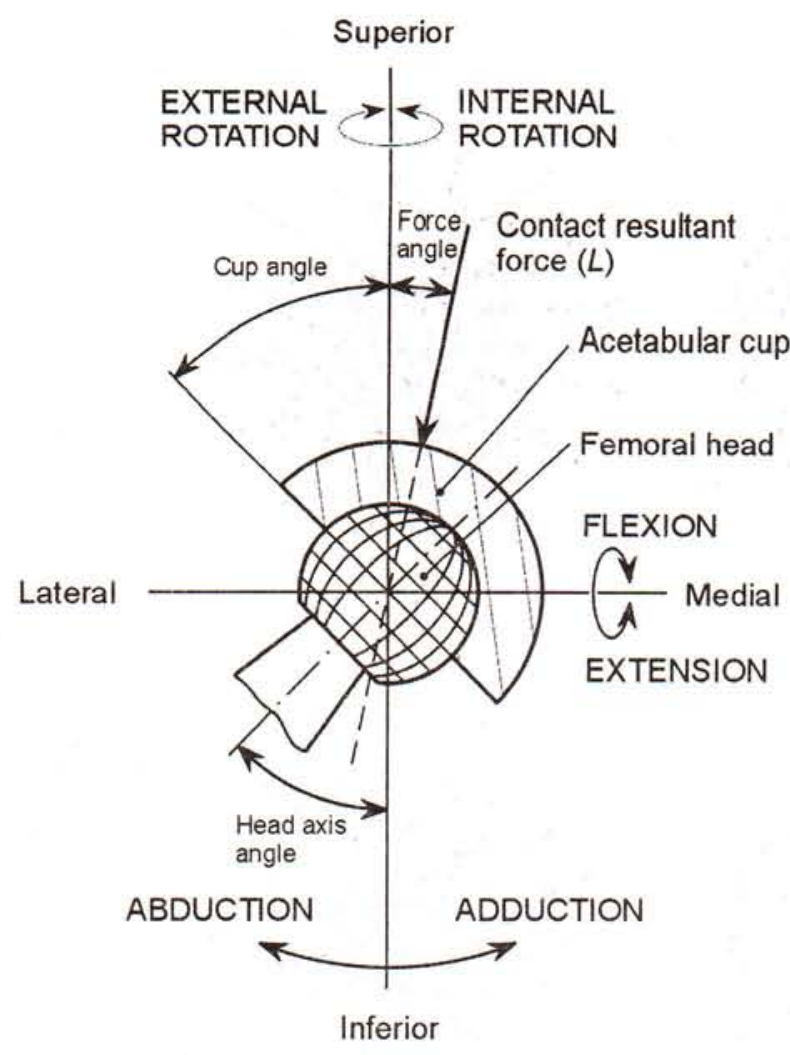

Fig. 1: Antero-posterior view of prosthetic joint with directions of motions shown (right hip, coronal plane)

$[5,20,21,22,23,24,25,26,27]$. The angles of all analyzed cases can be found in [28], Fig. 1 and [29], Fig. 1. The waveforms applied in the analyses of the BRM simulators are shown in Figs. $3 \mathrm{a}$ and $3 \mathrm{~d}$. The slide track computations were described in detail in [28] and [30].

For visualization, the tracks were plotted on a sphere representing the articular surface (Figs. $3 \mathrm{~b}$, e). Flattened tracks
[30] were plotted to give an overview of the whole pattern (Figs. 3c, f). In computing the slide tracks the number of marker points was not restricted, and the location of the points could be freely selected. Theoretically, there are an infinite number of tracks, but too many tracks on one plot would result in a tangled image. Therefore, a limited number of points were chosen to produce an illustrative track pattern.

The rotation sequence was determined according to the simulator design. The first rotation changed the orientation of both the other rotation axes of the simulator relative to the acetabular cup (the reference coordinate system). The second rotation changed the orientation of the one remaining simulator axis relative to the cup. The third rotation did not change the orientation of any simulator axis relative to the cup. In two-axis simulator designs, the first step in determining the rotations was naturally omitted. The sequence for gait was $\mathrm{FE} \rightarrow \mathrm{AA} \rightarrow$ IER, since the goniometer used by Johnston and Smidt [19] conformed to this sequence.

The computed slide tracks were verified experimentally. Head tracks were engraved in the HUT-3 and HUT-BRM simulators with sharp pins of hardened steel embedded in the acetabular cup ([28], Fig. 4). The pins were placed very carefully to produce an accurate pattern corresponding to the marker points in the computational model. The simulators were driven for one cycle with load on to produce the tracks. The lengths, locations and shapes of the engraved tracks were compared with the computed tracks.

The effect of the shape of the rotation-prevention lever of the BRM simulators on the head tracks was studied both by engraving the track grooves as explained above, and by letting a stationary pen draw tracks on the head while the simulator was driven without the cup ([29], Fig. 5). The cup tracks of the BRM-simulator were checked by photographing the movement of head marker points with long exposure times.

To be able to determine the wear factor one must compute the integral $\int L \mathrm{~d} x$ by considering the temporal variation of the
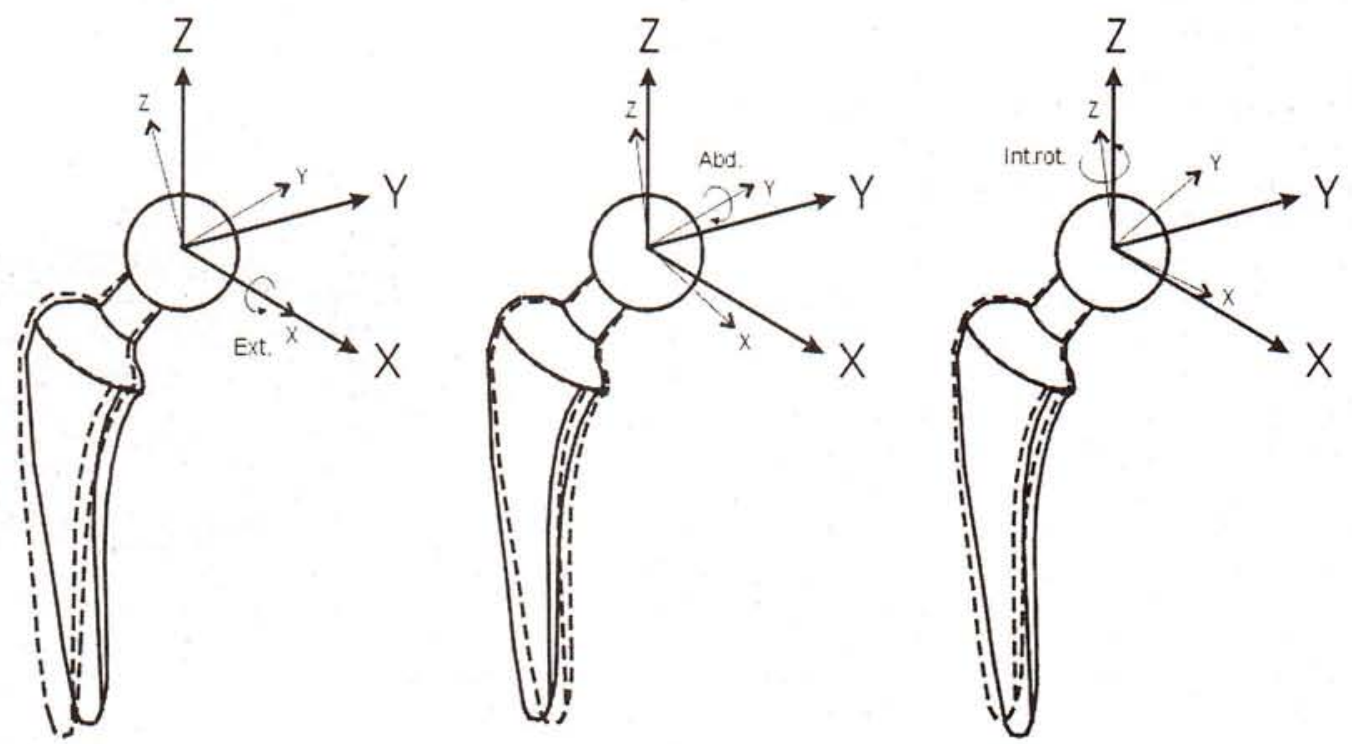

Fig. 2: Euler rotations applied to the right hip. Sequence is $\mathrm{FE} \rightarrow \mathrm{AA} \rightarrow \mathrm{IER}$. In the computations, positive rotations corresponded to extension, abduction and internal rotation. The reference coordinate system $X Y Z$ was fixed relative to the cup and the moving coordinate system $x y z$ was fixed relative to the head. 


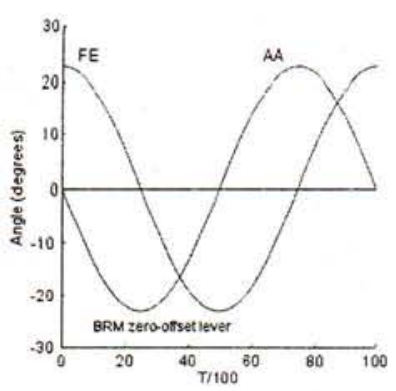

(a)

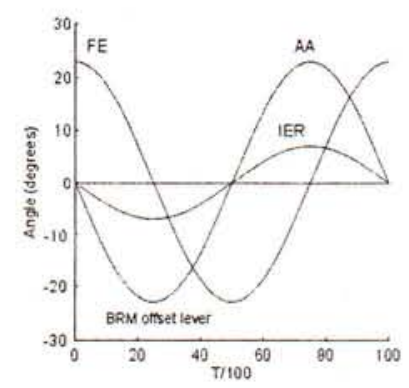

(d)

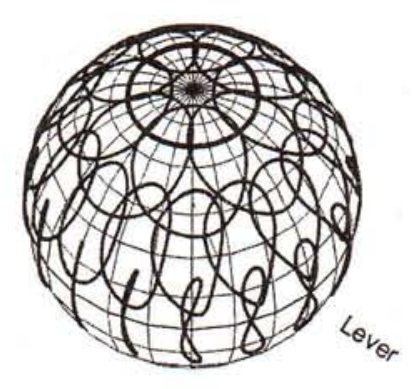

(b)

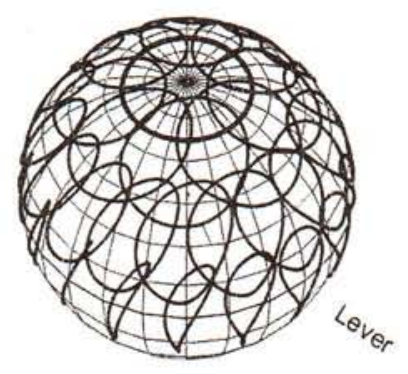

(e)

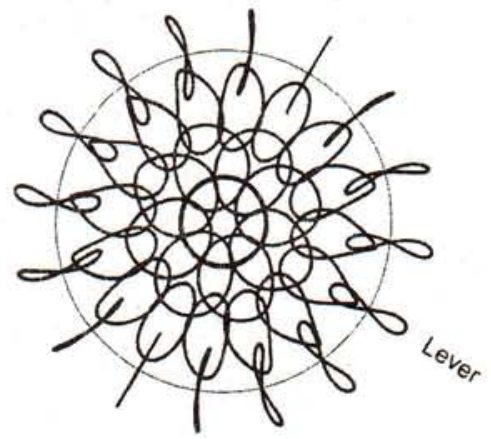

(c)

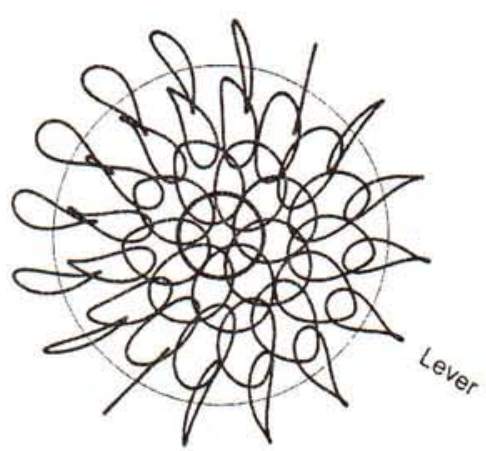

(f)

Fig. 3: Motion waveforms, slide tracks on femoral head and whole pattern of 41 flattened tracks of BRM hip wear simulators with direction of rotation prevention lever indicated. Note that diameter of circle is $\pi r$ (not $2 r$ ). Force track shown with thick line. (a)-(c) Waveforms (FE, AA) and tracks of HUT-BRM. (d)-(f) Waveforms (FE, AA, IER) and tracks of BRM simulator with offset lever.

contact resultant force $L$ along the force track, i.e. the track drawn by $L$ on the counterface (Fig. 4). In [31] this was done for eleven simulator designs and for level walking as measured by Johnston and Smidt [] by numerically integrating the product of the instantaneous load and increment of sliding distance over a cycle: $\int L \mathrm{~d} x=\sum L_{i} \Delta x_{i}$. The various load waveforms and peak values that were used in the computations can be found in [31]. The gait force track was a simplification in that the track was drawn by the resultant force in the position $12^{\circ}$ to the vertical in the XZ-plane. While the force track thus drawn was considered adequate for wear factor computations by corresponding to the area of highest contact pressure, fluctuations in the direction of the resultant force during the gait cycle have been reported [17]. The time step between adjacent points on the load and motion waveforms and consequently on the force track was $\Delta t_{i}=T / N$. Additional force track computations included the sliding distance $x_{L}=\sum \Delta x_{i}$, the sliding velocity $v=\Delta x_{i} / \Delta t_{i}$ and the direction of sliding relative to the cup $\xi$. The quantity $\xi$ was the direction of the velocity vector (and of the frictional shear stress) relative to its direction at $t=0$, and was indicated by the instantaneous tangent of the force track. The tangent was approximated by a line connecting two adjacent track points. The sum of the changes in the direction of sliding along the force track, $\Sigma\left|\Delta \xi_{i}\right|$, was computed.
To take into account the variation of relative motion and contact pressure all over the contact surface, the force track integral $\int L \mathrm{~d} x$ was replaced by a surface integral computed as a sum of track integrals [32]. The computation involved

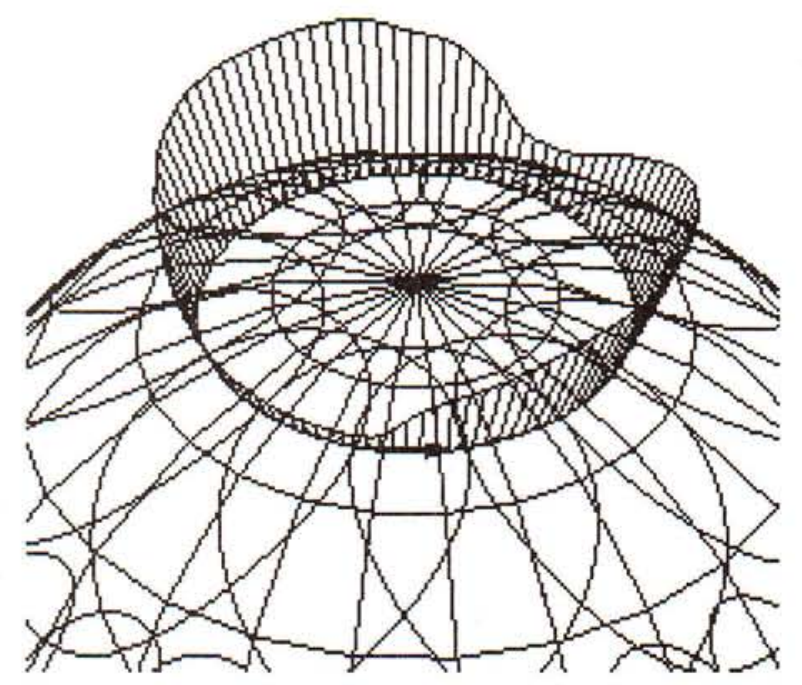

Fig. 4: Double-peak waveform of contact resultant force $L$ plotted along the force track of the HUT-BRM simulator 
replacing the contact pressure distribution with a large number of discrete forces and summing the integrals computed for each discrete force along their respective track. The discrete forces acted normal to the surface and represented the pressure on a small surface area element. Each integral was computed numerically by summing the product of the instantaneous force value and increment of sliding distance. This approach was tested for the HUT-BRM simulator in which the dynamic load had a double-peak waveform and an average value of $1.0 \mathrm{kN}$ and was applied along the vertical symmetry axis of the cup (Fig. 5a). For this simulator, wear test results were available [33] and the relative motion computations had been verified [28]. Next, a brief overview of the computations is given. A detailed presentation can be found in [32].

Because in the HUT-BRM the load was applied along the symmetry axis of the cup, the pressure distribution could be written as the product of the peak value and a shape function of the angle $\theta$ between the position vector and the $Z$-axis only

$$
p=p_{\max } f(\theta) \text {. }
$$

The shape functions of the ellipsoidal, paraboloidal and sinusoidal pressure distributions were $\sqrt{\left(1-4 \theta^{2} / \pi^{2}\right)}$, $1-\theta^{2} / \pi^{2}$ and $\cos \theta$, respectively. The corresponding values of

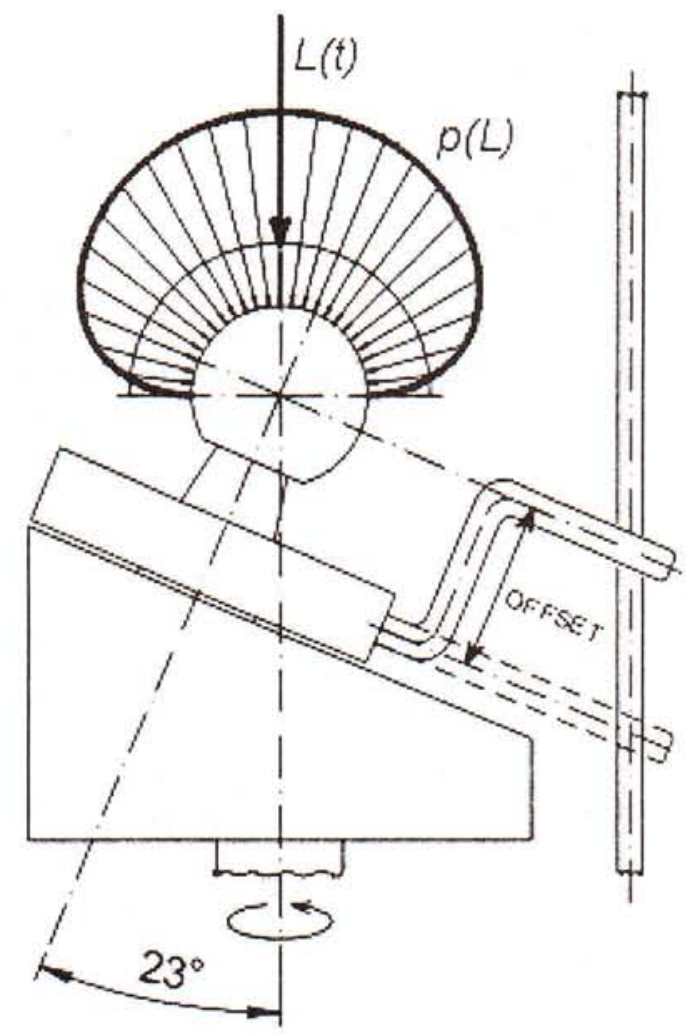

$p_{\max }$ were $0.386 L / r^{2}, 0.453 L / r^{2}$ and $0.477 L / r^{2}$. These values were determined by the vertical force equilibrium

$$
L=\int_{A} p \cos \theta \mathrm{d} A
$$

The wear tests had shown that during steady-state wear the area of contact was hemispherical during the whole load cycle. Therefore a pressure distribution extending to the rim of the cup and varying only in height during the cycle could be selected for the computation. A different wear test configuration would naturally result in a different pressure distribution as the angle between the direction of load and the symmetry axis of the cup significantly influences the shape of the distribution [34]. In the present case the effect of the shape of the pressure distribution was studied by selecting three commonly used distributions, ellipsoidal, paraboloidal and sinusoidal [35]. The hemispherical contact surface was divided into approximately equisized area elements (Fig. 5b), so that the distribution of the discrete forces would resemble the corresponding pressure distribution.

When the contact surface had been divided into surface elements $A_{\mathrm{E}, m}, m=1, \ldots, N_{\mathrm{E}}$, the discrete forces at each time step $t_{i}, i=1, \ldots, N$ were calculated as

$$
q_{m}\left(t_{i}\right)=p\left(\theta_{m}, t_{i}\right) A_{\mathrm{E}, m}
$$

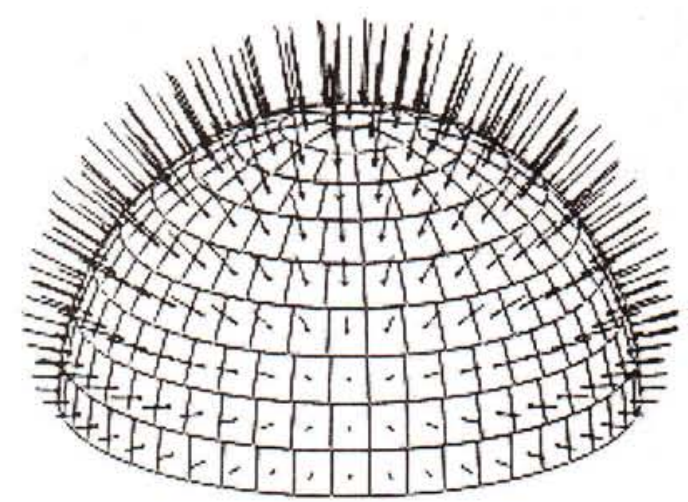

(a)

(b)

Fig. 5: (a) Schematic drawing of HUT-BRM simulator with ellipsoidal contact pressure distribution $p(L)$ varying with time due to load $L(t)$. Offset lever position shown with dashed lines. (b) Ellipsoidal contact pressure distribution discretized by large number of normal forces. Temporal variation of each force along its slide track was taken into account in computing an alternative for the integral $\int L \mathrm{~d} x$, the sum of track integrals $I$. 
The pressure value $p\left(\theta_{m}, t_{i}\right)$ was calculated in the middle of element $m$, which was also the point of application of $q_{m}\left(t_{i}\right)$. The slide tracks of the discrete forces were computed as described in [28]. Finally, the sum of track integrals $I$ was computed:

$$
I=\sum_{m=1}^{N_{E}} \oint q_{m} d x=\sum_{m=1}^{N_{E}} \sum_{i=1}^{N} q_{m}\left(t_{i}\right) \Delta x_{m}\left(t_{i}\right)
$$

\section{Results}

The gait slide tracks were mostly irregular ovals, having a characteristic thorn near the end of the gait cycle. Tracks with high aspect ratio and small track figures were also present (Fig. 6a). The HUT-3 slide tracks were mostly elliptical, but tracks with high aspect ratio and short tracks were present, so that the overall slide track pattern resembled the pattern produced by the gait simulation (Fig. $6 \mathrm{~b}$ ). The motion waveforms proposed in the ISO/DIS 14242-1 standard generated open slide tracks on the center of contact and an elliptical force track (Fig. 6c). The BRM slide tracks included a circle, egg and tear shapes, and figures of eight (Fig. 3). In this paper, only head tracks are shown. The cup tracks of the gait, HUT-BRM and HUT-3 simulations were presented in [28], Figs. 5, 6, 8. Both head and cup track patterns of eight contemporary hip simulators were shown in [29], Figs. 3a-h.

For the HUT-3 and BRM simulators, the shapes of the individual engraved tracks and the overall engraved track pattern agreed well with the computed tracks ([28], Figs. 6a, $7,8 \mathrm{a}$ and 9 ). The lengths of the engraved force tracks of the HUT-3 and the BRM simulators were $24.2 \mathrm{~mm}$ and $34.6 \mathrm{~mm}$, respectively for $r=14 \mathrm{~mm}$. The lengths of the corresponding computed force tracks were $23.9 \mathrm{~mm}$ and 34.3 $\mathrm{mm}$, differences being $-1.2 \%$ and $-0.9 \%$, respectively. Both the computed and engraved force tracks had aspect ratios of 1 in the BRM simulator and 3.8 in the HUT-3 simulator.

In the BRM simulator, changing the type of rotation-prevention lever changed the slide track pattern. The lever with offset caused tilting and shift of tracks, compared to the zero-offset case (Fig. 3). The force track however, remained unchanged. In [29], it was shown that a rotation-prevention lever with an offset introduced IER motion in the simulator, turning the BRM into a three-axis device and causing the change in slide track pattern. This experimental finding was reproduced in the computations by adding the IER motion to the BRM slide track simulation done previously ([29], Fig. 6).

The tangent to the slide track indicated the direction of sliding at any moment during the motion cycle. If the slide tracks were open loops or figures of eight, the direction of sliding changed continually implying multidirectional motion. A circular or elliptical slide track meant that the direction of sliding (and consequently the direction of the frictional shear stress) gradually changed $360^{\circ}$ during one cycle. When the aspect ratio of the track increased, the changes in the direction of sliding became more abrupt near the turning points of the track. Tracks with a very high aspect ratio or linear tracks suggested that the motion was reciprocating.

In contemporary hip simulators the length of the force track ranged from $1.41 r$ to $2.46 r$. In the gait simulation the length of the force track was $1.65 r$. With the common femoral head diameter of $28 \mathrm{~mm}$, the force track length ranged from 19.7 to $34.3 \mathrm{~mm}$ per cycle, the average sliding speed from 19.7 to $49.0 \mathrm{~mm} / \mathrm{s}$ and $/ L \mathrm{~d} x$ from 17.4 to $43.5 \mathrm{~N} \mathrm{~m}$ [31]. A summary of the results is given in Table 1 .

When the sum of track integrals $I$ was computed for the HUT-BRM simulator the value first decreased with an increasing number of elements [32]. However, the maximum decrease (which occurred for the ellipsoidal pressure distribution) was less than $0.14 \%$ when the number of elements was increased from 500 to 1000 . It was therefore considered that there was no need to increase the number of elements above 1000 . With dynamic load and 1000 discrete forces the values of $I$ and the sum of element forces for the ellipsoidal, paraboloidal and sinusoidal pressure distributions were $3.49 r$, $3.24 r, 3.20 r$ (units are $\mathrm{N} \mathrm{m}$ when $r$ is substituted in $\mathrm{mm}$ ) and $1.69 L, 1.53 L$ and $1.50 L$ respectively. The $\int L d x$ value in the resultant force case was $2.46 \mathrm{r} \mathrm{Nm}$.

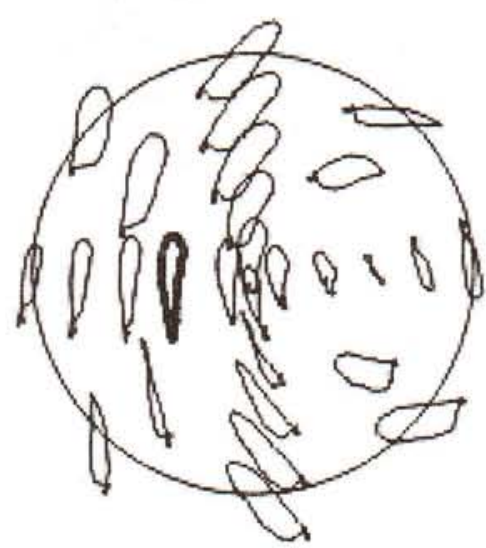

(a)

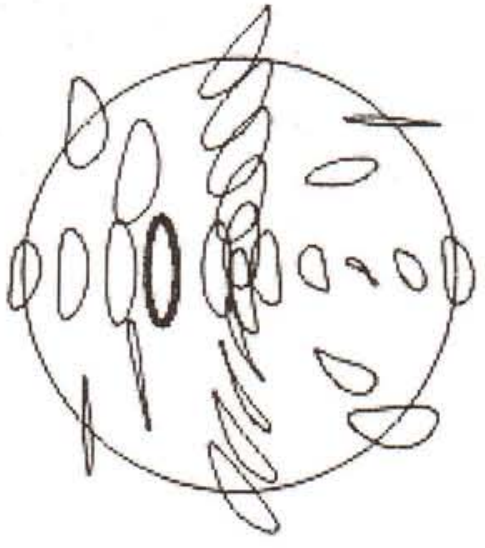

(b)

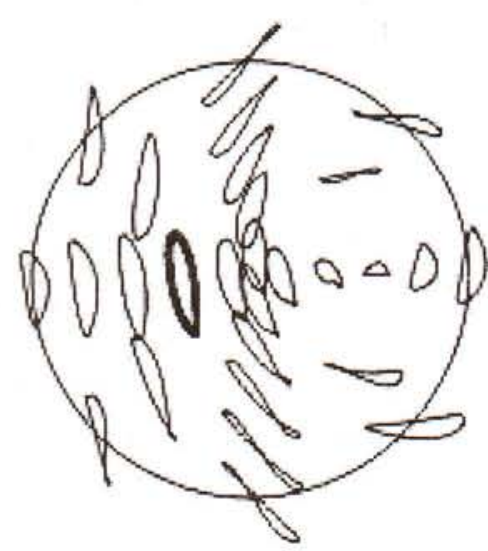

(c)

Fig. 6: Flattened slide track patterns of femoral head tracks. Thick line indicates force track. (a) Gait motion. (b) HUT-3 simulator. (c) ISO/DIS 14242-1 simulator specification, assuming sequence $\mathrm{FE} \rightarrow \mathrm{AA} \rightarrow \mathrm{IER}$. 
Table 1: Summary of force track computations for eleven simulators and gait

\begin{tabular}{|l|l|l|l|c|c|c|}
\hline \multicolumn{1}{|c|}{ Case } & $\begin{array}{c}\mathrm{T} \\
{[\mathrm{s}]}\end{array}$ & $\begin{array}{c}\mathrm{x}_{\mathrm{L}}{ }^{\mathrm{a}} \\
{[\mathrm{mm}]}\end{array}$ & $\begin{array}{c}v_{\text {mean }}{ }^{\mathrm{a}} \\
{[\mathrm{mm} / \mathrm{s}]}\end{array}$ & $\begin{array}{c}\left\langle L \mathrm{~d} x^{\mathrm{a}}\right. \\
{[\mathrm{Nm}]}\end{array}$ & $\begin{array}{c}\sum\left|\Delta \xi_{i}\right| \\
{[\mathrm{degrees}]}\end{array}$ & Reference \\
\hline HUT-3 & 0.85 & $1.71 r$ & $2.01 r$ & $3.10 r$ & 360 & {$[24]$} \\
\hline HUT-BRM $^{\mathrm{b}}$ & 0.98 & $2.46 r$ & $2.50 r$ & $2.46 r$ & 360 & {$[25]$} \\
\hline MMED-BRM $^{\mathrm{c}}$ & 0.88 & $2.46 r$ & $2.78 r$ & $2.00 r$ & 360 & {$[36]$} \\
\hline MTS-BRM ${ }^{\mathrm{c}}$ & 1.00 & $2.46 r$ & $2.46 r$ & $2.31 r$ & 360 & {$[8]$} \\
\hline AMTI & 0.50 & $1.75 r$ & $3.50 r$ & $1.33 r$ & 648 & {$[5]$} \\
\hline Munich & 1.00 & $1.67 r$ & $1.67 r$ & $1.48 r$ & 557 & {$[27,37]$} \\
\hline Leeds Mk I & 1.00 & $1.41 r$ & $1.41 r$ & $1.24 r$ & 433 & {$[21]$} \\
\hline ISO/DIS 14242-1 & 1.00 & $1.58 r$ & $1.58 r$ & $2.03 r$ & 364 & {$[23]$} \\
\hline Durham Mk II & 1.00 & $1.59 r$ & $1.59 r$ & $2.24 r$ & 380 & {$[26]$} \\
\hline Leeds Mk II & 1.00 & $1.57 r$ & $1.57 r$ & $1.35 r$ & 400 & {$[20]$} \\
\hline ProSim & 1.08 & $1.65 r$ & $1.52 r$ & $1.25 r$ & 1513 & {$[22]$} \\
\hline Gait & 1.13 & $1.65 r$ & $1.46 r$ & $1.59 r$ & 876 & {$[19,38]$} \\
\hline
\end{tabular}

${ }^{a} r$ to be substituted in $\mathrm{mm}$; ${ }^{\mathrm{b}}$ zero-offset lever; ${ }^{\mathrm{c}}$ offset lever

\section{Discussion}

In the hip joint, the direction of sliding between the femoral head and the acetabular cup changes continually during walking motion. In the slide track computations the continually changing direction of sliding manifested itself as open track figures. Notably, in the three-axis simulators and the purely biaxial HUT-BRM the force tracks were open shapes ([28], Figs. 5-6, 8 and [29], Figs. 3a-e, 4a-e, i). Biaxial simulators omitting the abduction-adduction motion, had linear or high aspect ratio force tracks ([29], Figs. 3f-h). Especially for polyethylene, multidirectional motion is a requirement for producing the amount of wear and the type of wear particles that correspond to clinical findings [7]. Therefore, continually changing direction of sliding must be considered a design requirement for simulators to be used for assessing polyethylene wear [8]. Linear, reciprocating motion has been shown to underestimate the wear rate, while exaggerating the effect of counterface roughness on the wear rate [9].

The slide track computations made it possible to visualize the relative motion at the articulation and to qualitatively compare different simulator designs with each other and with the slide track pattern pertaining to walking motion. But the software could additionally offer a basis for quantitative analysis of sliding distances, sliding speeds, the integral $\int L \mathrm{~d} x$ used in computing the wear factor $k$, and the changing direction of sliding during the motion cycle. It was also possible to propose an improved method of computing the wear factor, taking into account the variation in loading and motion on the whole contact surface of the prosthetic hip joint.

It should be noted that the computational approach presented was not just a slight modification of an earlier method [39]. It was a new systematic method of determining the motion of marker points attached to either the acetabular cup or the femoral head relative to the counterface. First, the Euler sequence was determined by investigating how a rotation about one axis changed the orientation of the other rotation axes relative to the cup [28]. Subsequently, the rotation angles were applied in accordance with the sequence. Furthermore, the method of computing the slide tracks was experimentally verified both by engraving patterns of tracks on the femoral head with hardened steel pins embedded into the acetabular cup and by drawing the tracks on the head with a stationary pen while the simulator was run for one cycle [28, 29]. The lengths and angular extents of the computed tracks were in good agreement with the corresponding values of these experimental tracks [30]. For the most popular hip wear simulator, the BRM, an experimentally verified slide track pattern was presented [28]. Additionally, it was shown that changing the shape of the rotation-prevention lever changed the slide track pattern due to the addition of IER motion [29]. The influence of the shape of the rotation-prevention lever can be seen in Fig. 3 and Figs. 9 and 10 in [28], and Figs. 5 and 6 in [29].

Because the essential features of walking motion are inevitably captured in the gait waveforms produced by recording hip joint motion during level walking [19], these waveforms were used to compute the reference slide track pattern. However, many of the gait tracks had a thorn towards the end of the motion cycle because of the sudden changes in FE and IER. It is unlikely that a simulator would need to duplicate these sudden changes that last only for a short period of the total motion cycle, since simulators with smooth circular force tracks (BRM, CTPOD) have been shown to produce realistic polyethylene wear $[9,25]$. If abrupt changes in directions of motion are incorporated in the design, the simulator could be plagued with annoying vibrations. To avoid this problem, the motion waveforms used in the HUT-3 simulator were idealizations of the gait waveforms measured by Johnston and 
Smidt [19]. Therefore, smooth slide tracks were produced while the overall track patterns for the gait and HUT-3 computations resembled each other (Fig. 6).

A factor which is more likely to influence the wear rate than small differences between motion waveforms is the aspect ratio of the force track. Under similar wear conditions (28 mm diameter polished $\mathrm{CoCr}$ head, serum lubricant and similar load) the wear rate produced by the BRM simulator was found to be twice that produced by the HUT-3 simulator [25]. In the BRM, the aspect ratio was 1 (circular force track), whereas in the HUT-3, it was 3.8 (elliptical force track). Reciprocating motion is known to result in minimal wear [6, 8]. Thus, an explanation for the difference in wear rate is that the motion producing an elliptical force track is closer to reciprocation than the motion producing a circular force track.

Hip simulators are usually designed so that the FE motion dominates in the high pressure region of the contact. In walking, the FE motion has the largest amplitude and will determine the maximum extent of the force track figure. In addition, the distances of the point of application of the resultant contact load to the rotation axes will emphasize the influence of FE and AA motions on the shape of the force track. The load is usually applied near the IER axis and consequently the distances to the $\mathrm{FE}$ and $\mathrm{AA}$ axes are much greater, which has the effect of diminishing the role of the IER motion in shaping the force track. The AA motion determines the extent of the force track transversely to the $\mathrm{FE}$ direction. The phase difference between the FE and AA motions determines how much the force track figure opens up. For example, an elliptical force track is produced with sinusoidal waveforms and a phase difference of $\pi / 2$ between $\mathrm{FE}$ and AA. In the high pressure region of the three-axis simulators, the dimensions of the slide tracks in the major and transverse directions were determined mainly by $\mathrm{FE}$ and $\mathrm{AA}$, which resulted in roughly elliptical slide tracks. In the two-axis simulators with the IER $\rightarrow \mathrm{FE}$ sequence, reciprocating motion with IER rotation superimposed was produced along the force track. Open slide track figures were produced as the distance from the marker point to the IER axis increased, such that the largest elliptical tracks were near the equator of the cup ([29], Figs. 3f, g). In the high pressure region of the BRM simulators, the FE motion did not dominate over the AA motion, and therefore the tracks had aspect ratios close to one.

The slide track software made it possible to investigate the effect of changing motion waveform amplitudes or phases, the Euler sequence, or of omitting certain waveforms to simplify the design of a simulator. For example, Fig. 7 shows how omitting either AA or IER waveforms changes the gait slide track pattern. If IER is left out, the narrow part of the force track and the other tracks in the middle of the pattern widens. Tracks near the equator and the vertical column of tracks in the middle become narrower. If AA is left out, the effect is nearly opposite. The force track and most of the tracks in the middle become narrow figures of eight, while the other tracks remain open loops. Consider designing a simulator starting from the gait waveforms and with the Euler sequence for gait. In the light of Fig. 7, simplifying the three-axis walking motion to two-axis motion (to limit manufacturing costs of the design) is probably better done by omitting IER than AA. Omitting AA would result in a force track of high aspect ratio which may influence the amount of wear produced by the simulator. As such, nearly reciprocating motion suggested by high aspect ratio tracks is known to underestimate the polyethylene wear rate compared to the clinical wear rate of polyethylene acetabular cups. In simulators with high aspect ratio tracks on the center of contact, lower wear in this region may be compensated for by higher wear towards the equator of the cup where open tracks were produced. The track pattern of biaxial simulators without AA motion can be contrasted with the pattern of the BRM simulator in which the open tracks are located on the center of contact and the narrow tracks and the figures of eight are located on the equator.

The $\int L \mathrm{~d} x$ integrals determined in the force track computations made it possible to convert the wear rates produced by different simulators into wear factors that provide a better basis for comparison between simulators and with clinical values. As an example [31], consider the highly differing wear rates of conventional gamma-sterilised polyethylene cups against polished $28 \mathrm{~mm}$ diameter $\mathrm{CoCr}$ heads with serum-based lubricant in the HUT-3 and AMTI simulators, 57 and $25 \mathrm{mg}$ per one million cycles, respectively [ 5,25$]$. The corresponding wear factors were equal, which can be verified by dividing the wear rate by the density, $0.94 \mathrm{mg} / \mathrm{mm}^{3}$, and by the corresponding $\int L \mathrm{~d} x$ integrals (Table 1 ):

$\left(57 \mathrm{mg} / 0.94 \mathrm{mg} / \mathrm{mm}^{3} / 10^{6}\right) /(3.10 \cdot 14 \mathrm{Nm})=1.4 \cdot 10^{-6} \mathrm{~mm}^{3} / \mathrm{Nm}$ and

$\left(25 \mathrm{mg} / 0.94 \mathrm{mg} / \mathrm{mm}^{3} / 10^{6}\right) /(1.33 \cdot 14 \mathrm{Nm})=1.4 \cdot 10^{-6} \mathrm{~mm}^{3} / \mathrm{Nm}$. These values can be compared with the mean clinical wear factor in Charnley prostheses with conventional polyethylene cups which was found to be $2.9 \cdot 10^{-6} \mathrm{~mm}^{3} / \mathrm{Nm}[15]$. The difference between the clinical and the simulator wear factors can be explained by the considerable scratching of the stainless steel femoral heads in vivo. The range in the clinical wear factors was as large as $0.09-7.2 \cdot 10^{-6} \mathrm{~mm}^{3} / \mathrm{Nm}$.

In Table 1 it can be seen that there were considerable variations between the different cases regarding the length of the force track $x_{\mathrm{L}}$. Additionally, the variations persisted when $x_{\mathrm{L}}$ was divided with the cycle time $T$ to give the mean sliding velocity along the force track. Most simulators appear to have approximately the same mean sliding velocity as that obtained for gait, except for the BRM simulators, in which it was 66-88 \% higher and one simulator in which it was as much as 2.4 times higher. If the velocity is significantly higher than in gait, there is a risk of altering the lubrication conditions and consequently the accuracy of the wear simulation. The cumulative change of direction of sliding, $\Sigma\left|\Delta \xi_{i}\right|$, can be seen as a measure of the unevenness or jerkiness of the force track. It could be anticipated that a higher value of $\Sigma\left|\Delta \xi_{i}\right|$ would indicate higher wear. However, even a small loop along the track causes the value of $\sum\left|\Delta \xi_{i}\right|$ to increase by $360^{\circ}$ while the increase in wear remains negligible due to the small sliding distance. Therefore it appears unnecessary to design a simulator based on very jerky and complicated motion waveforms, i.e., a simulator with a very high $\sum\left|\Delta \xi_{i}\right|$ value.

The conventional way of computing $\int L \mathrm{~d} x$ along the force track does not take into account the contact pressure distribution and the variation of relative motion with location on the contact surface. It also neglects the transverse pressure component which is perpendicular to the direction of the resultant load $L$. When the sum of track integrals $I$ was computed by replacing the pressure distribution with a large 


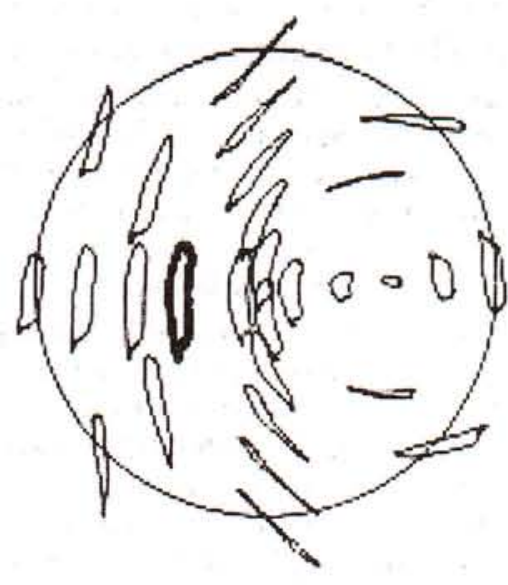

(a)

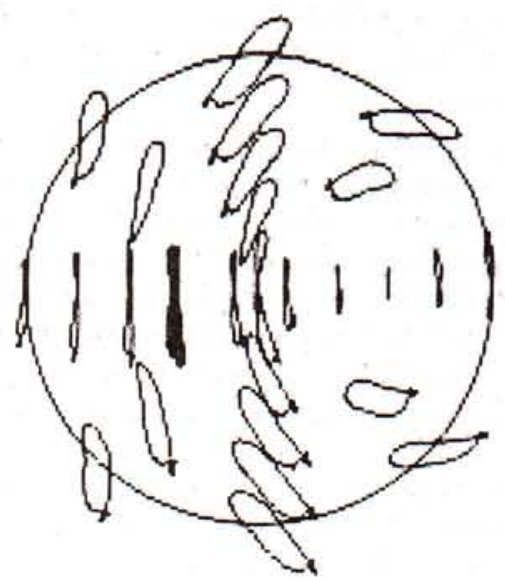

(b)

Fig. 7: Effect of omitting IER or AA motion on the gait slide track pattern drawn on the femoral head. (a) IER motion was omitted. Sequence was $\mathrm{FE} \rightarrow \mathrm{AA}$. (b) AA motion was omitted. Sequence was $\mathrm{FE} \rightarrow \mathrm{IER}$. Pattern shown in Fig. 6a includes all motion components.

number of discrete forces, the whole contact situation was better represented including components of transverse load and variation of sliding distance with location. However, the value of $I$ was substantially higher than the value of $\int L \mathrm{~d} x$ and consequently the wear factor was lower. For example, an average wear rate of $16.6 \mathrm{mg}$ per one million cycles was obtained with the HUT-BRM simulator for gamma-irradiated conventional polyethylene cups against polished $28 \mathrm{~mm}$ diameter $\mathrm{CoCr}$ heads, lubricant diluted calf serum, and test length 3 million cycles [33]. In the conventional way of calculating $k$, the result would be $16.6 \mathrm{mg} /\left(10^{6} \cdot 0.93 \mathrm{mg} / \mathrm{mm}^{3} \cdot 2.46 \cdot 14 \mathrm{Nm}\right)$ $=0.52 \cdot 10^{-6} \mathrm{~mm}^{3} / \mathrm{Nm}$. With an ellipsoidal contact pressure distribution and 1000 elements, the surface integral gave a value of $3.49 \cdot 14 \mathrm{Nm}=48.9 \mathrm{Nm}$. Hence, $k=0.36 \cdot 10^{-6} \mathrm{~mm}^{3} / \mathrm{Nm}$, which is $30 \%$ lower compared with the resultant force case [32]. It appears that computing the integral $\int L \mathrm{~d} x$ with a single resultant force substantially overestimates the wear factor. In fact, all wear factors published earlier for total hip prostheses are more or less overestimates, as they rely on the assumption of single point contact.

The method of replacing $\int L \mathrm{~d} x$ with $I$ requires that a reasonable estimate of the contact pressure distribution can be computed. The computation would vary from case to case because the angle between the direction of load and the symmetry axis of the cup influences the shape of the distribution [34]. The accuracy of the estimate of the distribution could be improved if additional factors such as the thickness and the material of the cup, the metal backing shell of the cup and the clearances between the head and the cup and between the shell and the cup could be taken into account.

Previous studies [39, 40] have presented slide tracks traced by marker points on the head, i.e., cup tracks. In [28] and [29], head tracks were presented for the first time. The head tracks show how points on the cup slide against the counterface, and make it possible to compute the sliding distances of differential area elements of the acetabular cup. The sliding of an area element of the cup can be compared to the sliding of the test pin against the counterface disk in the
CTPOD simulator. Recently, tests were done with a modified version of the CTPOD simulator in which the slide track of each pin had a different aspect ratio [41]. These tests showed that the wear factor of conventional polyethylene was proportional to $\mathrm{I} / \sqrt{\mathrm{AR}}$ and that for $\mathrm{AR}$ values exceeding 5.5 the wear factor decreased to unrealistically low values. Given the relationship $k=k(\mathrm{AR})$, an interesting future research topic would be to investigate whether these test results could be linked with the head track computations to relate the slide track pattern to the wear of the cup. A numerical measure of wear involving the variation of load, sliding distance and track aspect ratio on the bearing surface could, in principle, be computed by summing the wear rate contributions of each surface element, as shown in (5).

$$
\sum_{m=1}^{N_{\mathrm{E}}} k_{m} \oint q_{m} \mathrm{~d} x
$$

In (5), $k_{m}=k_{m}\left(\mathrm{AR}_{m}\right)$ is the wear factor which corresponds to the aspect ratio of the slide track of the discrete force $q_{m}$. The applicability of this approach needs to be verified with comparative wear tests, as the accuracy of the computation primarily depends on whether experimental wear data produced directly with pin-on-disk type devices can be used for computing wear rate predictions for ball-in-socket configurations. Another factor influencing the computation would be the accuracy of the estimation of the pressure distribution in the joint.

\section{Conclusions}

This paper treated the analysis of relative motion between the femoral head and the acetabular cup in the prosthetic hip and the relation between type of motion and wear. The slide tracks produced by walking motion and by contemporary hip simulators were computed. Computations important for wear assessment included the determination of the integral $\int L \mathrm{~d} x$ along the force track and subsequently the sum of track 
integrals of forces obtained by discretization of the contact pressure distribution. Based on the research, the following conclusions can be drawn:

1. The slide track software made it possible to visualize and analyze the relative motion of points on the articular surfaces of the hip joint. In addition, it was possible to investigate the effect of changing the Euler sequence or of omitting certain waveforms to simplify the design of a simulator. The effect of changes to the motion waveform amplitudes or phases could be investigated by only generating new waveforms, i.e., without large modifications to the software. By analyzing published data, it was possible to make a slide track overview of practically all contemporary hip simulators ([29] together with [28]). The slide track computations were experimentally verified with the HUT-BRM and the HUT-3 simulators. The gait tracks [28] and the tracks of other simulators [29] were computed according to the same principles as the verified tracks. The software was used to compute quantities relevant for assessing wear, such as the sliding speed or the cyclic variation of the direction of sliding [31].

2. For the BRM simulators, experimentally verified slide tracks were presented and it was shown that the shape of the rotation-prevention lever affected the slide track pattern ([28], Figs. 9 and 10). An offset rotation-prevention lever introduced IER motion, turning the simulator into a three-axis device [29].

3. The slide track overview showed that in the three-axis simulators (and the purely biaxial HUT-BRM) the force tracks were open shapes ([29], Figs. 3a-e; 4a-e, i). Biaxial simulators omitting the abduction-adduction motion had linear or high aspect ratio force tracks ([29], Figs. 3f-h). When the abduction-adduction motion was omitted in the gait computations (Fig. 7b), a similar effect was seen. Thus, the simulators can be divided into two groups: those that include and those that omit the AA-motion. Lower wear due to high aspect ratio tracks on the center of contact can perhaps be compensated for by increased wear due to open tracks elsewhere, especially if the contact pressure distribution extends to the equator of the cup. One can qualitatively compare simulators, e.g., by noting that under similar wear conditions, a higher force track aspect ratio implies less wear [25]. However, to be able to reliably quantify the relationship between the slide track pattern and wear, comparative tests are required to be done with the same type of specimens, lubricant and loading. Published wear test reports differ from each other regarding the materials, the type and dilution of serum lubricant, etc. and cannot be used for finding such a relationship.

4. Simulators built according to the ISO/DIS 14242-1 standard (assuming an $\mathrm{FE} \rightarrow \mathrm{AA} \rightarrow \mathrm{IER}$ rotation sequence) would produce a slide track pattern with an elliptical force track and other open slide tracks on the center of contact ([29], Fig. 3e), similar to the slide tracks present in the pattern of the HUT-3 simulator. The design requirement that the direction of sliding shall be continually changing would therefore be fulfilled by simulators meeting the ISO/DIS 14242-1 specifications. A similar slide track pattern was, however, produced with only the FE and AA components of the gait motion (Fig. 7a), which suggests that the simulator need not be three-axial to obtain wear conditions similar to those specified in ISO/DIS 14242-1.

5. The integral $\int L \mathrm{~d} x$ was evaluated for the most used contemporary hip simulator designs by discretizing the load curve and evaluating the integral along the force track. The integral makes it possible to compare wear test results between simulators and with clinical wear measurements in terms of the wear factor $k$.

6. The sum of track integrals $I$ was computed by discretizing the contact pressure distribution and summing the contributions of many normal forces distributed all over the bearing surface. For the HUT-BRM simulator the value of $I$ was substantially higher than the value of $\int L \mathrm{~d} x$, suggesting that the traditional way of computing the integral with a single resultant force overestimates the wear factor.

7. Wear tests with the slide track shape as the primary variable were recently done with a modified version of the CTPOD wear simulator [41]. The wear factor of conventional polyethylene was found to be proportional to $1 / \sqrt{\mathrm{AR}}$. Future research could elucidate the possibilities for linking this result with the slide track computations to find a quantitative relationship between the slide track pattern and the wear in the prosthetic joint.

\section{References}

[1] Nevalainen, J., Hirvonen, A., Pulkkinen, P.: The 1998-1999 Implant Yearbook on Orthopaedic Endoprostheses. Vantaa, National Agency for Medicines, 2000.

[2] Bauer, T. W., Schils, J.: The Pathology of Total Joint Arthroplasty II. Mechanisms of Implant Failure. Skeletal Radiol, Vol. 28, 1999, p. 483-497.

[3] Ingham, E., Fisher, J.: Biological Reactions to Wear Debris in Total Joint Replacement. Proc Inst Mech Engr (H), Vol. 214, 2000, p. 21-37.

[4] Revell, P. A., Al-Saffar, N., Kobayashi, A.: Biological Reaction to Debris in Relation to Joint Prosthesis. Proc Inst Mech Engr (H), Vol. 211, 1997, p.187-197.

[5] Bragdon, C. R., O'Connor, D. O., Lowenstein, J. D., Jasty, M., Syniuta, W. D.: The Importance of Multidirectional Motion on the Wear of Polyethylene. Proc Inst Mech Engr (H), Vol. 210, 1996, p. 157-165.

[6] Saikko, V.: A Multidirectional Motion Pin-on-disk Wear Test Method for Prosthetic Joint Materials. J Biomed Mater Res, Vol. 41, 1998, p. 58-64.

[7] Wang, A., Stark, C., Dumbleton, J. H.: Mechanistic and Morphological Origins of Ultra-high Molecular Weight Polyethylene Wear Debris in Total Joint Replacement Prostheses. Proc Inst Mech Engr (H), Vol. 210, 1996, p. 141-155.

[8] Wang, A., Polineni, V. K., Essner, A., Sokol, M., Sun, D. C., Stark, C., Dumbleton, J. H.: The Significance of Nonlinear Motion in the Wear Screening of Orthopaedic Implant materials. J Testing Eval, Vol. 25, 1997, p. 239-245.

[9] Wang, A., Essner, A., Polineni, V. K., Stark, C., Dumbleton, J. H.: Lubrication and Wear of Ultra-high Molecular Weight Polyethylene in Total Joint Replacements. Trib Int, Vol. 31, 1998, p. 17-33.

[10] Ahlroos, T.: Effect of Lubricant on the Wear of Prosthetic Joint Materials. Doctoral dissertation, Acta Polytechnica 
Scandinavica, Me 153, Helsinki, The Finnish Academies of Technology, 2001.

[11] Saikko, V.: Effect of Lubricant Protein Concentration on the Wear of Ultrahigh Molecular Weight Polyethylene. J Tribol, 2003 , in press.

[12] Saikko, V., Calonius, O., Keränen, J.: Effect of Counterface Roughness on the Wear of Conventional and Crosslinked Ultrahigh Molecular Weight Polyethylene Studied with a Multidirectional Motion Pin-on-disk Device. J Biomed Mater Res, Vol. 57, 2001, p. 506-512.

[13] Saikko, V., Calonius, O.: Wear of Conventional and Crosslinked Ultrahigh Molecular Weight Polyethylene Acetabular Cups Against Polished and Roughened CoCr Femoral Heads in a Biaxial Hip Simulator. J Biomed Mater Res Appl Biomater, Vol. 63B, 2002, p. 848-853.

[14] Saikko V., Keränen J.: Wear Simulation of Alumina-on-alumina Prosthetic Hip Joints Using a Multidirectional Motion Pin-on-disk Device. J Am Cer Soc, Vol. 85, 2002, p. 2785-2791.

[15] Atkinson, J. R., Dowson, D., Isaac, J. H., Wroblewski, B. M.: Laboratory Wear Tests and Clinical Observations of the Penetration of Femoral Heads into Acetabular Cups in Total Replacement Hip Joints. III: The Measurement of Internal Volume Changes in Explanted Charnley Sockets after 2-16 Years in Vivo and the Determination of Wear Factors. Wear, Vol. 104, 1985, p. 225-244.

[16] Bennett, D. B., Orr, J. F., Baker, R.: Movement Loci of Selected Points on the Femoral Head for Individual Total Hip Arthroplasty Patients Using Three-dimensional Computer Simulation. The Journal of Arthroplasty, Vol. 15, 2000, p. 909-915.

[17] Bergmann, G., Deuretzbacher, G., Heller, M., Graichen, F., Rohlmann, A., Strauss, J., Duda, G. N.: Hip Contact Forces and Gait Patterns from Routine Activities. Journal of Biomechanics, Vol. 34, 2001, p. 859-871.

[18] Morlock, M., Schneider, E., Bluhm, A., Vollmer, M., Bergmann, G., Müller, V., Honl, M.: Duration and Frequency of Every Day Activities in Total Hip Patients. Journal of Biomechanics, Vol. 34, 2001, p. 873-881.

[19] Johnston, R. C., Smidt, G. L.: Measurement of Hip-joint Motion During Walking-evaluation of an Electrogoniometric Method. J Bone Joint Surg, Vol. 51-A, 1969, p. 1083-1094.

[20] Barbour, P. S. M., Stone, M. H., Fisher, J.: A Hip Joint Simulator Study Using Simplified Loading and Motion Cycles Generating Physiological Wear Paths and Rates. Proc Inst Mech Engr (H), Vol. 213, 1999, p. 455-467.

[21] Dowson, D., Jobbins, B.: Design and Development of a Versatile Hip Joint Simulator and a Preliminary Assessment of Wear and Creep in Charmley Total Replacement Hip Joints. Engineering in Medicine, Vol. 17, 1988, p. 111-117.

[22] Goldsmith, A. A. J., Dowson, D.: A Multi-station Hip Joint Simulator Study of the Performance of $22 \mathrm{~mm}$ Diameter Zirconia-ultra-high Molecular Polyethylene Total Replacement Hip Joints. Proc Inst Mech Engr (H), Vol. 213, 1999, p. 77-90.

[23] ISO/DIS 14242-1 Draft International Standard, 2001: Implants for Surgery - Wear of Total Hip Joint Prostheses Part 1: Loading and Displacement Parameters for Wear-test- ing machines and Corresponding Environmental Conditions for Test.

[24] Saikko, V.: A Three-axis Hip Joint Simulator for Wear and Friction Studies on Total Hip Prosthesis. Proc Inst Mech Engr (H), Vol. 210, 1996, p. 175-185.

[25] Saikko, V., Ahlroos, T.: Type of Motion and Lubricant in Wear Simulation of Polyethylene Acetabular Cup. Proc Inst Mech Engr (H), Vol. 213, 1999, p. 301-310.

[26] Smith, S. L., Unsworth, A.: A Five-station Hip Joint Simulator. Proc Inst Mech Engr (H), Vol. 215, 2001, p. 61-64.

[27] Ungethüm, M.: Tribologisch-biomechanische Untersuchungen für den totalen Gelenkersatz der menschlichen Hüfte. Doctoral Dissertation. Rheinisch-Westfälische Technische Hochschule Aachen, 1976.

[28] Saikko, V., Calonius, O.: Slide Track Analysis of the Relative Motion Between Femoral Head and Acetabular Cup in Walking and in Hip Simulators. J Biomech, Vol. 35, 2002, p. $455-464$.

[29] Calonius, O., Saikko, V.: Slide Track Analysis of Eight Contemporary Hip Simulator Designs. J Biomech, Vol. 35, 2002, p. 1439-1450.

[30] Calonius, O.: Tribology of Prosthetic Joints - Validation of Wear Simulation Methods. Doctoral dissertation, Acta Polytechnica Scandinavica, Me 159, Helsinki, The Finnish Academies of Technology, 2002.

[31] Calonius, O., Saikko, V.: Force Track Analysis of Contemporary Hip Simulators. J Biomech, 2003, in press.

[32] Saikko, V., Calonius, O.: An Improved Method of Computing the Wear Factor for Total Hip Prostheses Involving the Variation of Relative Motion and Contact Pressure with Location on the Bearing Surface. J Biomech, 2003, in press.

[33] Saikko, V., Calonius, O., Keränen, J.: Effect of Extent of Motion and Type of Load on the Wear of Polyethylene in a Biaxial Hip Simulator. J Biomed Mater Res Part B: Appl Biomater, Vol. 65B, 2003, p. 186-192.

[34] Ipavec, M., Brand, R. A., Pedersen, D. R., Mavčič, B., Kralj-Iglič, V., Iglič, A.: Mathematical Modelling of Stress in the Hip During Gait. Journal of Biomechanics, Vol. 32, 1999, p. 1229-1235.

[35] Fricker, D. C.: Friction When Femoral Prosthesis Heads Slide in Acetabular Cups. In: "Ceramics in Substitutive and Reconstructive Surgery." (Ed. Vincenzini, P.). Amsterdam: Elsevier Science Publishers, 1991, p. 207-215.

[36] McKellop, H. A., Clarke, I. C.: Degradation and Wear of Ultra-high-molecular-weight Polyethylene. In: "Corrosion and Degradation of Implant Materials: Second Symposium, ASTM STP 859." (Eds. Fraker, A. C., Griffin, C. D.). Philadelphia: American Society for Testing and Materials, 1985, p. 351-368.

[37] Walter, A.: Investigations on the Wear Couple Biolox Forte Biolox Forte and Earlier Alumina Materials. In: "Performance of the Wear Couple Biolox Forte in Hip Arthroplasty" (Ed. Puhl, W.). Stuttgart: Ferdinand Enke Verlag, 1997, p. 123-135.

[38] Paul, J. P.: Forces Transmitted by Joints in the Human Body. Proceedings of the Institution of Mechanical Engineers, Vol. 181, Part 3J, 1967, p. 8-15.

[39] Ramamurti, B. S., Bragdon, C. R., O'Connor, D. O., Lowenstein, J. D., Jasty, M., Estok, D. M., Harris, W. H.: 
Loci of Movement of Selected Points on the Femoral Head During Normal Gait. The Journal of Arthroplasty, Vol. 11, 1996, p. 845-852.

[40] Ramamurti, B. S., Estok, D. M., Jasty, M., Harris, W. H.: Analysis of the Kinematics of Different Hip Simulators Used to Study Wear of Candidate Materials for the Articulation of Total Hip yrthroplasties. Journal of Orthopaedic Research, Vol. 16, 1998, p. 365-369.

[41] Saikko, V., Calonius, O., Keränen, J.: Effect of Slide Track Shape on the Wear of Ultra-high Molecular Weight Polyethylene in a Pin-on-disk Wear Simulation of Total Hip Prosthesis. J Biomed Mater Res Part B: Appl Biomater, 2003, in press.

Olof Calonius, Dr.Tech.

phone: +35894514573

fax: +35894513418

e-mail: Olof.Calonius@hut.fi

Helsinki University of Technology

Department of Mechanical Engineering

P.O. Box 4400

FIN-02015 HUT, Finland

Vesa Saikko, Dr.Tech.

phone: +35894513562

fax: +35894513542

e-mail:Vesa.Saikko@hut.fi

Helsinki University of Technology

Department of Mechanical Engineering

P.O. Box 4300

FIN-02015 HUT, Finland 